\title{
A NILPOTENT LIE ALGEBRA WITH NILPOTENT AUTOMORPHISM GROUP
}

\author{
BY JOAN L. DYER
}

\author{
Communicated by L. Auslander, August 21, 1969
}

Recent work of Stein, Knapp, Koranyi and others has been concerned with nilpotent Lie groups which admit expanding automorphisms, that is, semisimple automorphisms whose eigenvalues are all greater than one in absolute value (cf. [1], [3], [5]). It was an open question whether all nilpotent Lie groups admit expansions.

We first present a result due to Louis Auslander (oral communication) which establishes the existence of a class of Lie groups which do admit expanding automorphisms (\$1). We then present an example of a nine dimensional Lie group which does not have an expanding automorphism (\$2). In our work it is more convenient to use $\mathrm{Lie}$ algebra language than Lie group language. As is well known, for connected simply connected nilpotent Lie groups the choice of group or algebra language is a matter of taste.

I wish to take this opportunity to thank Louis Auslander for suggesting this problem to me.

1. Let us begin by recalling some definitions. Details may be found in [2, Chapter V] or in [6, Chapter 5]. Henceforth algebra will mean algebra over the reals.

We say that $\mathbb{E}$ is a free Lie of algebra rank $r$ if there exist $r$ elements $X_{1}, \cdots, X_{r} \in \mathfrak{\&}$ which generate $\mathfrak{\&}$ qua algebra and which enjoy the following universal mapping property: any function from the set $\left\{X_{1}, \cdots, X_{r}\right\}$ to any algebra $\mathscr{L}$ extends to a unique algebra homomorphism $\mathfrak{E} \rightarrow \mathfrak{L}$.

Define the ideals $\mathfrak{L}^{i}, i=1,2, \cdots$ of $\mathscr{E}$ as follows:

$$
\mathfrak{L}^{1}=\mathfrak{L}, \quad \mathfrak{L}^{i+1}=\left[\mathfrak{L}^{i}, \mathfrak{L}\right] .
$$

An ideal $\mathfrak{g}$ of $\mathbb{E}$ is homogeneous if the vector space $\mathfrak{g}$ is isomorphic to the direct sum of $\mathscr{g} \cap \mathfrak{d}^{i} / \mathscr{G} \cap \mathfrak{d}^{i+1}, i=1,2, \ldots$. We shall say that the Lie algebra $\mathscr{E}$ is homogeneous if $\mathscr{L}$ is isomorphic to $\mathbb{E} / \mathfrak{g}$ with $\mathscr{E}$ free and $g$ homogeneous.

AMS Subject Classifications. Primary 2250, 2280; Secondary 1730, 2022.

Key Words and Phrases. Nilpotent Lie group, nilpotent Lie algebra, expanding automorphism. 
TheOrem. Any homogeneous Lie algebra admits expanding automorphisms.

Proof. We shall construct an expanding automorphism $\alpha: \mathfrak{L} \rightarrow \mathfrak{L}$ and show $\alpha(g) \subset g$. Let $\lambda$ be any real number of absolute value greater than one. Let $X_{1}, \cdots, X_{r}$ be free generators for $\boldsymbol{\mathcal { L }}$. It follows that the vector space spanned by the $X_{i}$ is of dimension $r$ and is a compliment of $\mathfrak{L}^{2}$. Set $\alpha\left(X_{i}\right)=\lambda X_{i}$, and extend $\alpha$ to an automorphism of $\mathfrak{\&}$ by the universal mapping property.

There is a basis of $\mathfrak{L}$ consisting of monomials in the $X_{\boldsymbol{i}}$, that is, bracket products of the $X_{\boldsymbol{i}}$. A monomial $\mu$ is of degree $d$ if $\mu \in \mathfrak{d}^{d}$ $-\mathfrak{S}^{d+1}$; equivalently, $\mu$ is nonzero and is a product involving precisely $d$ of the $X_{i}$, counting multiplicity. As $\alpha$ is a homomorphism, $\alpha(\mu)=\lambda^{d} \mu$. Hence $\alpha$ acts as scalar multiplication by $\lambda^{d}$ on the vector space $\mathcal{F}^{d}$ spanned by the monomials of degree $d$. Now $\mathcal{F}^{d}$ is isomorphic to $\mathfrak{L}^{d} / \mathfrak{L}^{d+1}$ under the map which sends each monomial to its coset modulo $\mathfrak{L}^{d+1}$, and we may decompose $\mathfrak{F}^{d}=L^{d} \oplus H^{d}$, where $H^{d}=\mathfrak{F}^{d} \cap \mathfrak{g}$. Since $g$ is homogeneous, it is the direct sum of its subspaces $H^{d}$, and therefore $\alpha(\mathfrak{g}) \subseteq \mathscr{g}$. Thus $\alpha$ induces an automorphism $\beta: \mathfrak{L} \rightarrow \mathfrak{L}$. But $\mathfrak{L}=\mathfrak{L} / \mathfrak{g}$ is isomorphic to the direct sum of $\mathcal{F}^{d} / \mathcal{F C}^{d} \cap \mathscr{g} \cong L^{d}$, so $\beta$ restricted to $L^{d}$ is scalar multiplication by $\lambda^{d}$. Thus all eigenvalues of $\beta$ are greater than one in absolute value, or, $\beta$ is expanding.

2. We recall that a nilpotent Lie algebra of class $c$ is an algebra for which $\mathscr{L}^{c} \neq 0, \mathscr{L}^{c+1}=0$ where the $\mathscr{L}^{i}$ are defined as above. Nilpotent algebras are algebra generated by any elements which generate $\mathfrak{L} / \mathfrak{L}^{2}$. A free nilpotent algebra of rank $r$ and class $c$ may be viewed as the quotient $\mathfrak{L} / \mathfrak{L}^{c+1}$, where $\mathfrak{L}$ is free of rank $r$. We note that any elements which form a basis of $\mathfrak{\&} / \mathfrak{\&}^{2}$, when viewed as elements of $\mathfrak{d} / \mathfrak{d}^{c+1}$ enjoy the universal mapping property for maps into nilpotent algebras of class at most $c$.

We now denote by $\mathscr{E}$ the free nilpotent Lie algebra of rank 2 and class 6 . We select a monomial basis $X_{1}, X_{2}, \cdots, U_{8}, U_{9}$ of $\&$ such that

$$
\begin{aligned}
X_{1}, X_{2} & \text { is a basis of } \mathfrak{L}_{\bmod \mathfrak{L}^{2},}, \\
V & \text { is a basis of } \mathfrak{L}^{2} \bmod \mathfrak{L}^{3}, \\
W_{1}, W_{2} & \text { is a basis of } \mathfrak{L}^{3} \bmod \mathfrak{L}^{4}, \\
Y_{1}, Y_{2}, Y_{3} & \text { is a basis of } \mathfrak{L}^{4} \bmod \mathfrak{L}^{5}, \\
Z_{1}, \cdots, Z_{6} & \text { is a basis of } \mathfrak{L}^{5} \bmod \mathfrak{L}^{6}, \\
U_{1}, \cdots, U_{9} & \text { is a basis of } \mathfrak{L}^{6} .
\end{aligned}
$$




\begin{tabular}{|c|c|c|c|c|}
\hline $\mathscr{L}$ & $X_{1}$ & $X_{2}$ & $\boldsymbol{V}$ & $W_{1}$ \\
\hline$X_{2}$ & $-\boldsymbol{V}$ & \multirow[b]{2}{*}{$W_{2}$} & \multirow[b]{3}{*}{$Z_{5}-Z_{4}$} & \\
\hline$V$ & $W_{1}$ & & & \\
\hline$W_{1}$ & $\boldsymbol{Y}_{3}$ & $Y_{2}$ & & \\
\hline$W_{2}$ & $\boldsymbol{Y}_{2}$ & $Y_{1}$ & $Z_{3}-Z_{2}$ & $2 U_{5}-U_{4}-U_{6}$ \\
\hline$Y_{1}$ & $Z_{2}$ & $Z_{1}$ & $U_{3}-U_{2}$ & \\
\hline$Y_{2}$ & $Z_{4}$ & $Z_{3}$ & $U_{5}-U_{4}$ & \\
\hline $\boldsymbol{Y}_{\mathbf{3}}$ & $Z_{6}$ & $Z_{5}$ & $U_{8}-U_{7}$ & \\
\hline$Z_{1}$ & $2 U_{2}-U_{3}$ & $U_{1}$ & & \\
\hline$Z_{2}$ & $3 U_{4}-3 U_{5}+U_{6}$ & $U_{2}$ & & \\
\hline $\boldsymbol{Z}_{3}$ & $U_{4}$ & $U_{3}$ & & \\
\hline$Z_{4}$ & $U_{7}$ & $U_{5}$ & & \\
\hline$Z_{5}$ & $U_{8}$ & $U_{6}$ & & \\
\hline$Z_{6}$ & $U_{9}$ & $2 U_{8}-U_{7}$ & & \\
\hline
\end{tabular}

FIGURE 1

With respect to this basis, the multiplication table of $\boldsymbol{E}$ is given in Figure 1. Unlisted products are obtained by antisymmetry or are zero; the entry in row $\mathrm{A}$ column $\mathrm{B}$ is the product $[A B]$.

Let $\mathscr{g}$ be the ideal of $\mathscr{L}$ generated by the set

$$
S=\left\{Y_{1}-Y_{3}, Z_{4}, Z_{1}-U_{4}, Z_{2}-3 Z_{3}-U_{4}\right\} .
$$

To obtain a vector space basis for $\mathscr{g}$, we use the fact that $g$ is generated by the union of the sets $S_{i}, i=1,2,3$ where

$$
S_{1}=S, \quad S_{i+1}=\left\{\left[W X_{j}\right]: j=1,2 ; W \in S_{i}\right\} .
$$

We remark that $S_{4}=0$ since $\left[\mathfrak{L}^{i}, \mathfrak{S}^{j}\right] \subset \mathfrak{L}^{i+j}$ and $S \subset \mathfrak{L}^{4}$. Thus $\mathscr{g}$ has basis:

$$
\begin{gathered}
Y_{1}-Y_{3}, Z_{1},-U_{4}, \quad Z_{2}-3 Z_{3}-U_{4}, \quad Z_{2}-Z_{6}, \quad Z_{4}, Z_{5}-U_{4}, \\
U_{1}, \quad U_{2}, \quad U_{3}, \quad U_{5}, \quad U_{6}, \quad U_{7}, \quad U_{8}, \quad U_{9}-3 U_{4} .
\end{gathered}
$$


Set $\mathfrak{L}=\mathfrak{L} / \mathfrak{g}$, and let $\pi: \mathfrak{L} \rightarrow \mathfrak{L}$ be the canonical projection. We have that $\mathfrak{L}$ is 9 -dimensional and has basis

$$
\begin{aligned}
& X_{1}=\pi X_{1}, \quad X_{2}=\pi X_{2}, \quad V=\pi V, \quad W_{1}=\pi W_{1}, \quad W_{2}=\pi W_{2}, \\
& Y_{1}=\pi Y_{1}, \quad Y_{2}=\pi Y_{2}, \quad Z=\pi Z_{3}, \quad U=\pi U_{4} .
\end{aligned}
$$

We shall now show that each automorphism of $\mathscr{L}$ is unipotent. It then follows from a theorem of E. Kolchin [4] that the group of automorphisms is nilpotent.

Let us first observe that if the automorphism $\alpha: \mathfrak{L} \rightarrow \mathfrak{L}$ induces the identity automorphism of $\mathscr{L} / \mathscr{L}^{2}$, it follows that $\alpha$ induces identity automorphisms of $\mathfrak{L}^{i} / \mathfrak{L}^{i+1}$ for each $i=1, \cdots, 6$ and therefore that $\alpha$ is unipotent. We must establish that $\alpha\left(X_{i}\right)=X_{i} \bmod \mathscr{L}^{2}, i=1,2$. However it does not suffice to consider $\alpha\left(X_{i}\right) \bmod \mathfrak{L}^{2}$ for the ideal $\mathscr{g}$ is not homogeneous.

We consider therefore

$$
\begin{aligned}
& \alpha\left(X_{1}\right)=a_{1} X_{1}+a_{2} X_{2}+a_{3} V \bmod \mathfrak{L}^{3}, \\
& \alpha\left(X_{2}\right)=b_{1} X_{1}+b_{2} X_{2}+b_{3} V \bmod \mathfrak{L}^{3} .
\end{aligned}
$$

We shall use the notation

$$
\lambda_{i j}=a_{i} b_{j}-a_{j} b_{i}, \quad i, j=1,2,3
$$

where $\lambda=\lambda_{12} \neq 0$ as $\alpha$ induces an automorphism of $\mathscr{L} \bmod \mathfrak{L}^{2}$.

One computes that

$$
\begin{aligned}
\alpha(V)= & {\left[\alpha X_{1}, \alpha X_{2}\right]=\lambda V+\lambda_{31} W_{1}+\lambda_{32} W_{2} \bmod \mathfrak{L}^{4} } \\
\alpha\left(W_{1}\right)= & {\left[\alpha V, \alpha X_{1}\right]=a_{1} \lambda W_{1}+a_{2} \lambda W_{2}+\left(a_{1} \lambda_{31}+a_{2} \lambda_{32}\right) Y_{1} } \\
& +\left(a_{2} \lambda_{31}+a_{1} \lambda_{32}\right) Y_{2} \bmod \mathfrak{L}^{5} \\
\alpha\left(W_{2}\right)= & {\left[\alpha V, \alpha X_{2}\right]=b_{1} \lambda W_{1}+b_{2} \lambda W_{2}+\left(b_{1} \lambda_{31}+b_{2} \lambda_{32}\right) Y_{1} } \\
& +\left(b_{2} \lambda_{31}+b_{1} \lambda_{32}\right) Y_{2} \bmod \mathfrak{L}^{5}
\end{aligned}
$$

which implies therefore that

(1) $\alpha(U)=\left[\alpha W_{1}, \alpha W_{2}\right]=\lambda^{3} U=\left[\alpha W_{1}, \alpha V\right]=-2 a_{2} \lambda^{2} Z \bmod \mathfrak{L}^{6}$ and so we have

$$
a_{2}=0, \quad \lambda=a_{1} b_{2} .
$$

Simplifying our computations accordingly we now find

$$
\begin{aligned}
\alpha\left(Y_{1}\right) & =\left[\alpha W_{1}, \alpha X_{1}\right]=\stackrel{a_{1}^{2} \lambda Y_{1}+3 a_{1}^{2} \lambda_{31} Z \bmod \mathcal{L}^{6}}{ } \\
& =\left[\alpha W_{2}, \alpha X_{2}\right]=\left(b_{1}^{2}+b_{2}^{2}\right) \lambda Y_{1}+2 b_{1} b_{2} \lambda Y_{2} \bmod \mathfrak{L}^{5} .
\end{aligned}
$$


Thus $b_{1} b_{2}=0, \lambda=a_{1} b_{2} \neq 0$ so

$$
b_{1}=0, \quad a_{1}^{2}=b_{2}^{2} .
$$

Now use (3) to obtain

$$
\alpha(U)=\left[\alpha Y_{1}, \alpha X_{2}\right]=a_{1}^{2} b_{2} \lambda U=a_{1} \lambda^{2} U
$$

and (1) then implies that $a_{1} \lambda^{2}=\lambda^{3}$ or

$$
b_{2}=1, \quad \lambda=a_{1} \text {. }
$$

Lastly, $\left[Y_{1}, X_{1}\right]=3 Z+U=3\left[Y_{2}, X_{2}\right]+U$; and equations (1) and (3) yield

$$
\left[\alpha Y_{1}, \alpha X_{1}\right]=3 \lambda^{2} Z+\lambda^{2} U, \quad 3\left[\alpha Y_{2}, \alpha X_{2}\right]+\alpha U=3 \lambda^{2} Z+\lambda^{3} U
$$

hence $\lambda^{2}=\lambda^{3}$ and so

$$
\lambda=a_{1}=1 .
$$

Equations (2), (4), (5) and (6) then state that $\alpha$ does induce the identity on $\mathscr{L} / \mathscr{L}^{2}$.

We remark that the construction of $\mathscr{L}$ and the computations of this section are valid over any field whose characteristic is not 2 or 3 .

ADDED IN PROOF. It has been brought to the attention of the author that the nilpotent Lie algebra constructed by J. Dixmier and W. G. Lister (Derivations of nilpotent Lie algebras, Proc. Amer. Math. Soc. 8 (1957), 155-158) has no expanding automorphisms. This algebra is shown to have a niplotent derivation algerba, which implies that the eigenvalues of any automorphism are roots of unity. The automorphism group of this algebra is however not nilpotent.

\section{REFERENCES}

1. D. B. A. Epstein and M. Shub, Expanding endomorphisms of flat manifolds, Topology 7 (1968), 139-141. MR 37 \#3580.

2. N. Jacobson, Lie algebras, Interscience, New York, 1962. MR $26 \# 1345$.

3. A. W. Knapp and E. M. Stein, Singular integrals and the principal theory (to appear).

4. E. Kolchin, On certain concepts in the theory of algebraic matric groups, Ann. of Math. (2) 49 (1948), 774-789. MR 10, 232.

5. A. Koranyi, I. Vagi and G. V. Welland, Remarks on the Cauchy integral and the conjugate function in a generalized halfplane (to appear).

6. W. Magnus, A. Karrass and D. Solitar, Combinatorial group theory. Presentations of groups in terms of generators and relations, Interscience, New York, 1966. MR 34 \#7617.

Lehman College, City University of New York, Bronx, New York 10468 and Institute for Advanced Study, Princeton, New Jersey 08540 